\section{Netzwerk DVAG}

\section{Liebes DVAG-Mitglied,}

wie Sie wissen, hat es sich der Verband $\mathrm{zu}$ einer seiner zentralen Aufgaben gemacht, ein dichtes Netzwerk zwischen den ihm angeschlossenen Berufsgeographinnen und -geographen zu knüpfen. Groß und nahezu unüberschaubar ist jedoch die Vielfalt an Arbeitsfeldern und Branchen, in denen Verbandsmitglieder tätig sind. Um transparenter $\mathrm{zu}$ machen, wo potenzielle Ansprechpartnerinnen und -partner zu finden sind, gibt es an dieser Stelle einen „Marktplatz“ mit Informationen zum geographischberuflichen Leben der Mitglieder. Wir möchten Sie daher herzlich bitten, der Redaktion berufliche Veränderungen, besondere - etwa wissenschaftliche - Leistungen und Auszeichnungen, Berufungen etc. mitzuteilen. Auch Angaben zu personellen Veränderungen auf der Ebene der Arbeitskreise und Regionalforen sind wünschenswert, ebenso können Eintritte in den Verband aufgenommen werden. Bitte schicken Sie ein Foto in druckfähiger Auflösung mit.

UCB

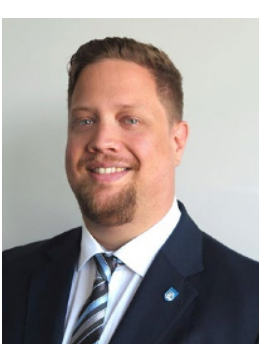

Swen Christian. (Foto: Gemeinde Wachtberg/mm)

Im Wintersemester 2012 hat Swen Christian an der Rheinischen Friedrich-Wilhelms-Universität zu Bonn seinen Abschluss als Diplom-Geograph gemacht. Der Titel der Diplomarbeit lautete ,Hochwasserschutz in der Bundesstadt Bonn - Konsequenzen aufgrund des Klimawandels“. Ergebnisse der Arbeit waren u.a. die Darstellung potenzieller räumlicher Konflikte und die Aufdeckung weiteren Forschungsbedarfs zum Thema.

Seit Januar 2018 ist Swen Christian Beigeordneter der Gemeinde Wachtberg. In dieser Funktion ist er Mitglied des Verwaltungsvorstands, allgemeiner Vertreter des Bürgermeisters und leitet das Dezernat „Technische Dienste“ mit den Fachbereichen Gemeindeentwicklung und Bauleitplanung sowie Infrastruktur und Bauen. Weiterhin ist ihm die Stabstelle IT und Digitalisierung zugeordnet. Inhaltlich ist er verantwortlich für die Themen Siedlungs- und Gewerbeflächenentwicklung, Städtebauförderung und Dorferneuerung, Hochwasserrisikomanagement, Umwelt-, Natur- und Klimaschutz, Mobilitätsmanagement und Digitalisierung. Außerdem ist er als Vorsitzender des Verwaltungsrats der Gemeindewerke Wachtberg AöR und Mitglied des Aufsichtsrats der enewa $\mathrm{GmbH}$ - Energie + Wasser Wachtberg verantwortlich für die Beteiligungen der Gemeinde. Über eine Kontaktaufnahme und fachlichen Austausch würde er sich freuen.

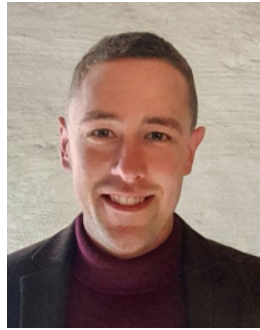

Thomas Hoyer

Ing. Thomas Hoyer M.Sc., 29 Jahre, ist seit Juli 2020 Leiter des Bauamts der Großen Kreisstadt Zschopau im mittleren Erzgebirge. Er hat Anfang 2017 sein Geographie-Studium an der TU Dresden mit dem Schwerpunkt Stadt- und Regionalplanung abgeschlossen und arbeitete seither im Bereich der ländlichen Entwicklung (LEADER Förderung) und in der Umweltplanung in einem Planungsbüro und in der Verwaltung. Die Leitung des Bauamts fordert ein breites Spektrum an Fähigkeiten und Methoden, die er durch das Geographie-Studium erlangen konnte. Die Stadtplanung und Stadtentwicklung sind dabei Kernaufgaben, weiterhin die Koordinierung und das Controlling kommunaler Hoch- und Tiefbaumaßnahmen, Akteursansprachen, Fördermittelakquise und die Bearbeitung baurechtlicher Angelegenheiten. Besonderes Augenmerk legt Thomas Hoyer dabei auf ökologische und soziale Nachhaltigkeit im Rahmen aller kommunalen Planungen.

Nebenbei ist Thomas Hoyer freiberuflich bundesweit als FLL-zertifizierter Baumkontrolleur und Baumgutachter tätig, wodurch er seine Passion für Bäume zur Profession gemacht hat.

Kontakt: geo_ing@web.de.

Kontakt: swen.christian@wachtberg.de. 


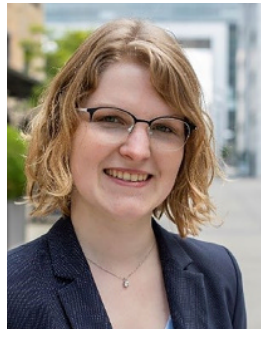

Anne Koller

M.Sc. Anne Koller hat in Münster und Köln Geographie mit den Schwerpunkten Öffentliches Recht sowie Stadt- und Regionalentwicklung studiert. Ihren Master schloss sie am 31. März dieses Jahres ab. Von April bis Juli absolvierte sie anschließend einen Freiwilligendienst beim BUND in Möggingen am Bodensee. Dort betreute sie Schutzgebiete und half bei der Kartierung seltener und gefährdeter Pflanzen. Seit 15. Juli 2020 unterstützt sie den Oberbergischen Kreis im Bereich Mobilität und stellt im Zuge des Förderprogramms „MobilitätsWerkStadt2025“ ein kreisweites, zukunftsfähges Mobilitätskonzept auf.

Anne Koller interessiert sich für die Bereiche Innenstadtentwicklung und Mobilitätsmanagement, aber auch für Naturschutz- und Umweltthemen. Sie ist schon lange Mitglied im DVAG und freut sich auch weiterhin auf angeregte Diskussionen und einen guten fachlichen Austausch-

Kontakt: anne.koller@aol.com.

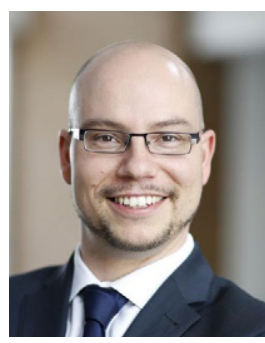

Stephan Mühl

Dipl.-Geogr. Stephan Mühl studierte an der TU Dresden Wirtschafts- und Sozialgeographie und ist seit 2019 bei der SIGNA Real Estate Management Germany GmbH tätig. Als Se- nior Portfolio Analyst im Bereich Department Stores ist er für das aktuelle Warenhaus-Portfolio der SIGNA in Deutschland verantwortlich. Das Kaufhof-Portfolio begleitet ihn schon seit 2012 aus der Zeit als Portfolio Manager Deutschland bei der METRO PROPERTIES (Immobiliengesellschaft der METRO AG) und der Deals von METRO AG zu Hudson's Bay Company bis hin zur österreichischen SIGNA Gruppe.

In seiner Tätigkeit ist er für die Aufbereitung, Analyse und das Management der Portfoliodaten sowie der Standort- und Marktanalysen aus der (handels-)immobilienspezifischen Sicht und Real Estate Research zuständig. Des Weiteren berät er das SIGNA Innovations Team in der Bewertung von GeoLocal Intelligence Start-Ups und nutzt die Erfahrung zur Ausschreibung von Softwaretools und Umsetzung von Prozessdigitalisierungen aus der Tätigkeit bei CORPUS SIREO als Project Manager IT und der METRO AG als GIS Specialist.

Seine berufliche Passion sind die Anwendungen und Nutzungen von Geodaten im vernetzten Kontext und deren Visualisierungs- und Analysemöglichkeiten.

Kontakt: s.muehl@signa.de.

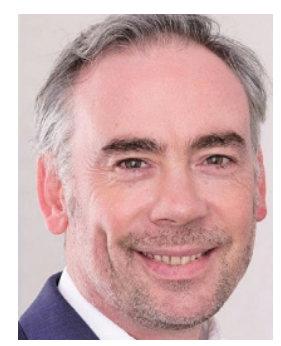

Thomas Schulz

Dipl. Geogr. M.Sc. Thomas Schulz verstärkt seit 1. Oktober 2020 das Kompetenzteam Einzelhandel \& Gastronomie im Unternehmensservice der KölnBusiness WirtschaftsförderungsGmbH am Börsenplatz in Köln.

Zuvor war er über 13 Jahre bei der REWE Markt GmbH, Zweignieder- lassung West, für die Supermarkt-Expansion im Köln-Bonner Raum tätig und unter anderem für die Neuansiedlung inkl. Umbau und Erweiterung bestehender REWE-Märkte zuständig.

Nach seinem Studium der Geographie in Köln und des Städtebaus in Bonn hatte Thomas Schulz ein immobilienwirtschaftliches Zusatzstudium in Wuppertal und Reading (UK) absolviert (REM-CPM) und als Einzelhandelsgutachter, Projektentwickler und Standortplaner unter anderem bei Globus, Praktiker und Edeka gearbeitet.

Kontakt: thomas.schulz@koeln.business.

\section{Jahre Standort- entwicklung an der Universität Augs- burg}

\section{Konstruktiver Partner des DVAG}

Die Fachgruppe Standortentwicklung am Institut für Geographie der Universität Augsburg feiert ihr zehnjähriges Bestehen. Im Wintersemsester 2009/2010 wurde erstmals im Studiengang Geographie das Nebenfach Standortentwicklung angeboten, das sich seither großer Beliebtheit bei den Studierenden erfreut. Inhaltlich werden anwendungsorientierte und praxisnahe Vorlesungen, Projektseminare und Exkursionen zu Themen wie Orts-, Stadt- und Regionalentwicklung, Wirtschaftsförderung, Tourismus, Konsumverhalten, Place Branding oder Arbeitsmarkt-Management angeboten. Mittlerweile ist diese Vertiefungsrichtung sowohl im Bachelorwie auch im Masterstudium ein fester Bestandteil des Lehrstuhls für Humangeographie und Transformationsforschung. Die Praxisnähe und Aktualität der Inhalte wird auch durch zahlreiche Lehrbeauftragte aus Wirtschaft und Verwaltung garantiert.

Die Fachgruppe Standortentwicklung leitet seit Beginn PD Dr. Markus 
Hilpert. Zu den aktiven Mitgliedern am Institut gehören zudem Christina Graß und Niklas Völkening. In ihren Forschungsprojekten $\mathrm{zu}$ regionalen Innovationsstrategien, Smart Cities oder zum innerstädtischen Einzelhandel kooperieren sie nicht nur eng mit Partnern aus der regionalen Ökonomie und der kommunalen Praxis.

Einen intensiven Austausch pflegt die Fachgruppe auch mit dem Regionalforum Schwaben des DVAG. Gemeinsam mit Dr. Bernhard Kräußlich und dem schwäbischen DVAG-Team organisiert sie beispielsweise jährlich die Martinitagung in Augsburg. Bei dieser Fachkonferenz diskutieren bis zu 200 Praktikerinnen und Praktiker über aktuelle Trends der Standortentwicklung, wie etwa den Nutzen intelligenter Technologien und künstlicher Intelligenz für Kommunen oder den Einsatz von social media im Standortmarketing.

Aktuell evaluiert sich die Fachgruppe Standortentwicklung, um auch für die kommenden zehn Jahre Strategien zur erfolgreichen Weiterentwicklung ihrer Forschung und ihres Studienangebots zu erarbeiten. Und sie freut sich auf zehn weitere Jahre der konstruktiven Partnerschaft mit dem DVAG.

Markus Hilpert

\section{Deutscher Kongress für Geographie:}

\section{\#GeoWoche2021 anstelle des DKG 2021}

Nach längerer, reiflicher Überlegung hat das Präsidium der Deutschen Gesellschaft für Geographie (DGfG) gemeinsam mit dem Ortsausschuss Frankfurt am Main beschlossen, den Deutschen Kongress für Geographie (DKG), der 2021 in Frankfurt stattfinden sollte, auf September $2023 \mathrm{zu}$ verlegen. „Wir bedauern die Notwendigkeit, diesen Schritt angesichts eines auch in Deutschland wieder zunehmenden Covid-19-Infektionsgeschehens setzen zu müssen. Herbst und Winter 2020/21 wären eine eminent wichtige Zeit für die Organisation eines Deutschen Kongresses für Geographie 2021, doch in der Pandemie erschienen die Planungsrisiken für diese Monate unverhältnismäßig hoch, die Unwägbarkeiten zu groß“, heißt es aus dem Präsidium. Die derzeitige Situation lasse daher keine andere Wahl.

In Zusammenarbeit mit den Teilverbänden wird die DGfG als Ersatz für den DKG 2021 die digitale \#GeoWoche2021 organisieren, die voraussichtlich vom 5 . bis 8 . Oktober 2021 stattfinden wird. ,Wir freuen uns auf Ihre vielfältige und kreative Unterstützung!“, richtet sich Prof. Dr. Werner Gamerith, Präsident der DGfG, an die gesamte deutschsprachige Geographie.

Im „Ortsausschuss“ der \#GeoWoche2021 wird der DVAG durch Dr. Maike Dziomba vertreten. Die Leiterin der DVAG-Geschäftsstelle hatte sich bereits im Ortsausschuss des Deutschen Kongresses für Geographie 2019 in Kiel kompetent und würdig für den DVAG und die Belange der Angewandten Geographie eingesetzt. Ihr Stellvertreter in diesem Gremium wird Prof. Dr. Henning Nuissl sein.

RED

\section{Aus Foren und Arbeitskreisen}

\section{Verstärkung für den AK Verkehr!}

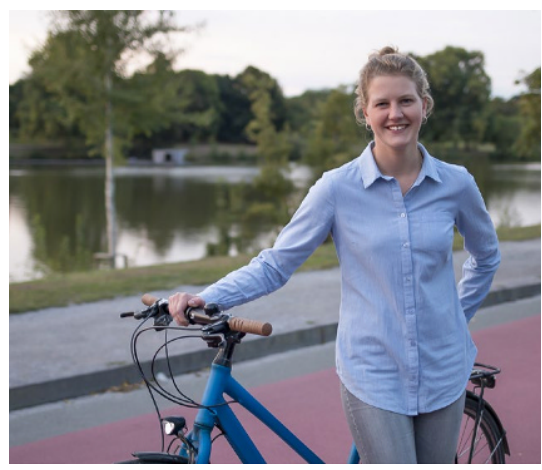

Annika Schröder

Wie bereits bei der DVAG-Jahrestagung am 11. September 2020 angekündigt, werde ich künftig als neue AK-Sprecherin für den AK Verkehr fungieren. Ich habe in Münster Humangeographie (M.Sc.) studiert und mich auch im Rahmen studienbegleitender Tätigkeiten stark mit nachhaltiger Mobilität, insbesondere Fahrradmobilität, beschäftigt.

Seit zwei Jahren arbeite ich nun bei der Stadt Münster im Fahrradbüro, das strategische und konzeptionelle Aufgaben sowie Öffentlichkeitsarbeit im Zusammenhang mit Radverkehr übernimmt.

Die Mobilität von heute und morgen gestalte ich mit viel Begeisterung. Diese möchte ich gerne in unseren Berufsverband hineintragen und zum Austausch mit interessierten Geograph*innen anregen. Ich freue mich, wenn der AK Verkehr künftig für einen fachlichen Austausch zwischen Mitgliedern aus Praxis und Wissenschaft genutzt werden kann. Bei Interesse an einer Mitarbeit im AK freue ich mich über eine Kontaktaufnahme. Weitere Informationen zur Gestaltung des AK Verkehr folgen demnächst.

Kontakt: SchroederA@stadt-muenster.de. 


\section{Regelmäßige Veranstaltungen der DVAG-Regionalforen}

\section{Berlin, jeden zweiten Dienstag im Monat, 19.00 Uhr}

Stammtisch des DVAG-Forums Berlin-Brandenburg-Mecklenburg-Vorpommern. Zurzeit ausgesetzt. Sobald ein neuer Treff gefunden ist, wird dieser im DVAG-Newsletter veröffentlicht und wird den Mitgliedern des Regionalforums mitgeteilt.

\section{Bonn/Rhein-Sieg/Ahrweiler, jeden zweiten Donnerstag im Monat}

Regelmäßiger Stammtisch des Regionalforums in Bonn. Mitglieder werden vorab per E-Mail über den Treffpunkt informiert. Wer noch nicht im regionalen E-Mail-Verteiler ist, kann sich gern an die Sprecher/innen wenden. Kontakt: Julia Fuchs, Jan Grade.

\section{Erlangen-Nürnberg, jeden zweiten Donnerstag im Monat, 18.30 Uhr}

Regelmäßiger Geographen-Stammtisch des Regionalforums ErlangenNürnberg in wechselnden Locations in Nürnberg und Erlangen. Mitglieder und weitere Interessierte werden ca. zwei Wochen vorab per E-Mail und auf der Facebookseite des Forums über den Ort informiert. Wer noch nicht in den regionalen E-Mail-Verteiler aufgenommen wurde, kann sich gerne an den Forumssprecher Simon Reichenwallner wenden.

\section{Frankfurt, jeden letzten Mittwoch im Monat, 19.00 Uhr}

Stammtisch des DVAG-Forums Rhein-Main in Frankfurt zum informellen Austausch, meist in einem zentral gelegenen Café bzw. Restaurant. Dem „Einkehrschwung“ geht oft ein kleiner ,urbanistischer Spaziergang" zu Themen wie Stadtplanung oder Immobilienmarkt voraus. Über den jeweils aktuellen Treffpunkt und die geplante Unternehmung wird eine Woche vorher mittels E-Mail-Verteiler des DVAG-Forums Rhein-Main sowie in der DVAG-Gruppe auf XING informiert. Falls möglich, wird um eine vorherige Anmeldung gebeten, spontane Gäste sind dennoch willkommen. Fragen und Rückmeldungen an: Silvia Beck, Verena Schmieg.

\section{Hamburg, jeden ersten Mittwoch im Monat, 20.00 Uhr}

Regelmäßiger Stammtisch des Forums Hamburg, wechselnde Locations. Mitglieder werden jeweils über den neuen Ort benachrichtigt. Kontakt: Andreas Obersteg, Jan Kotonski.

\section{Köln, jeden zweiten Mittwoch im Monat, 20.00 Uhr}

Regelmäßiger Jour fixe des Forums Köln, wechselnde Kneipen. Mitglieder werden per E-Mail über den Treffpunkt informiert. Kontakt: Stefan Hilger.

\section{München, jeweils am 13. der ungeraden Monate}

Das DVAG Regionalforum München stellt sich neu auf - jede Unterstützung ist willkommen, alle Interessierten sind herzlich eingeladen, dabei mitzuwirken! Mitglieder des DVAG Regionalforums München erhalten einen monatlichen Newsletter. Darin wird über Neuigkeiten, Termine und Stellenanzeigen aus München und der Region berichtet.

Darüber hinaus findet alle zwei Monate der Geographie-Treff statt, ein ungezwungener Austausch für alle. Wir treffen uns jeweils am 13. der ungeraden Monate eines Jahres: 13. September, 13. November usw. in wechselnden Locations. Über das nächste
Treffen informieren wir per Newsletter.

Kontakt: Luise Schnell, dvag-muenchen@ gmx.de.

\section{Münster}

Der DVAG-Stammtisch Münster trifft sich in wechselnden Lokalitäten. Stammtischtermine und Örtlichkeiten werden über den Regionalverteiler bekannt gegeben oder können bei den Forumssprecherinnen Birgit SchulzeRoberg und Jana Werring erfragt werden.

\section{Stuttgart, jeden ersten Dienstag im Monat, 19.00 Uhr}

Stammtisch des Forums Stuttgart, wechselnde Kneipen. Ca. zweimal jährlich findet der Stammtisch in Tübingen statt. Mitglieder werden per E-Mail über den Treffpunkt informiert. Interessenten können nachfragen unter wuerttemberg@geographiedvag.de. Kontakt: Thomas Holy.

Die Kontaktdaten der Forumssprecherinnen und -sprecher finden Sie am Ende des Heftes in der Rubrik „Organe des Verbandes“.

\section{Geplante Veranstaltungen}

Angesichts der Coronakrise sind allerorten die Veranstaltungsprogramm - nicht nur die des DVAG - gehörig durcheinandergeraten. Vieles wurde verschoben, zum Teil auf unbestimmte Zeit. Andere, ursprünglich als Präsenzveranstaltungen geplante Termine ließen sich jedoch durchaus erfolgreich in Online-Formate umswitchen. Um das Verbandsgeschehen weiter lebendig zu halten, wollen wir daran gern weiter festhalten. Wir freuen uns daher sehr, wenn sie Ideen und Anregungen für Veranstaltungen $\mathrm{zu}$ interessanten Themen in den Verband 
hineintragen. bei der Umsetzung unterstützen wir Sie gern.

Bitte wenden Sie sich mit Ihren Vorschlägen an die DVAG-Geschäftsstelle (Tel.: 04532/503 81 79; info@ geographie-dvag.de, https://www. geographie-dvag.de). Dort können Sie sich auch über den Stand der beiden nachstehenden Veranstaltungen informieren.

Darüber hinaus lohnt sich ein regelmäßiger Besuch der DVAG-Website auf jeden Fall. Da viele Online-Termine relativ kurzfristig angeboten werden, hält Sie ein Klick auf den Button „Veranstaltungen“ stets auf dem Laufenden!

\section{Voraussichtlich 20./21. März 2021, Bonn}

\section{DVAG-Sprecherworkshop 2021}

Wie jedes Jahr möchte der Vorstand alle Sprecher und alle interessierten Mitglieder zum Sprecherworkshop einladen, dies Mal wieder nach Bonn. Wir werden uns neben dem intensiven Austausch zwischen den Forenund Arbeitskreissprechern mit dem Vorstand vertieft mit ausgewählten Themen der Verbandsarbeit beschäftigen und gemeinsam über die $\mathrm{Zu}$ kunft des DVAG diskutieren. Zudem kann jeder Teilnehmer auch eigene Themenvorschläge einbringen. Der Sprecherworkshop soll eine lebendige Plattform zum Austauschen und Vernetzen sein. Die Teilnahme (inkl. Verpflegung, Übernachtung im EZ, nach Wunsch DZ) ist kostenlos; Reisekosten werden erstattet (sollte die Anfahrt für den Samstagvormittag zu lang sein, übernimmt der DVAG auch die Übernachtungskosten für Freitagnacht, damit alle Sprecher die Möglichkeit haben, anzureisen). Die Reise bitte erst nach Teilnahmebestätigung Ende Januar buchen.

Zeit: 20./21. März 2021, Samstag 13.00 Uhr bis Sonntag 13.00 Uhr. Ort: Bonn, Haus Venusberg, Haager Weg 28-30, 53127 Bonn. Der Link zur Anmeldung wird Anfang des Jahres 2021 auf der DVAG-Website freigeschaltet. Dort bei Interesse bitte bis zum 22. Januar 2021 anmelden (https://geographie-dvag.de/events/ sprecherworkshop-2021/).

\section{Ursprünglich geplant für 22. Juni 2020, Erlangen $\Rightarrow$ verschoben auf 2021}

\section{Praxisforum Geographie "Wirtschaftsförderung"}

Beraten, Netzwerken, Projekte managen, Veranstaltungen organisieren diese Aufgaben und viele mehr gehören zum vielseitigen Arbeitsalltag von Wirtschaftsförderer*innen. Dabei bieten unter anderem öffentliche Verwaltungen, Wirtschaftsverbände oder die Privatwirtschaft spannende Tätigkeitsbereiche für Geograph*innen.

Das Praxisforum „Wirtschaftsförderung" dient der Berufsorientierung von Geographie-Studierenden, wobei die Referent*innen Auskunft über ihren Berufseinstieg, ihren Praxisalltag und ihre Karriereplanung geben. Darüber hinaus erhalten auch Berufstätige sowie Lehrer*innen und Schulklassen interessante Einblicke in die geographische Praxis.

Anschließend bietet sich die Gelegenheit mit den Expert*innen in Kontakt zu treten und von ihren beruflichen Erfahrungen zu profitieren.

Das Praxisforum ist eine Veranstaltung des DVAG-Regionalforums Erlangen/Nürnberg in Kooperation mit der Alumni-Arbeit der Fränkischen Geographischen Gesellschaft und der Fachschaftsinitiative Geographie. Der Eintritt ist frei. Keine Anmeldung erforderlich. Veranstaltungsort: Friedrich-Alexander-Universität Erlangen-Nürnberg, Hörsaal C, Kochstraße 4, 91054 Erlangen. Moderation und Ansprechpartnerin: Manuela Burkert, E-Mail: manuela. burkert@geographie-dvag.de. 